\title{
The Development of Modern Chinese Animation Film Recording Technology
}

\author{
Yingman Liu \\ Conservatory of Music, Heze University, Heze, China \\ Email:yizhiyu997@163.com
}

Received 14 July 2015; accepted 14 August 2015; published 17 August 2015

Copyright @ 2015 by author and Scientific Research Publishing Inc.

This work is licensed under the Creative Commons Attribution International License (CC BY). http://creativecommons.org/licenses/by/4.0/

(c) (i) 0pen Access

\begin{abstract}
Modern Chinese animation film is accompanied by recording the continuous development of technological innovation, from the original subtitles instead of the sound development of the contemporary surround sound. The Chinese animation film recording technology has made great progress. The first recorded animation film of China has been introduced in this paper, for example, analyzing the recording and the early late, the influence of noise and the application of stereo, so as to provide reference for animation filmmakers and related practitioners.
\end{abstract}

\section{Keywords}

\section{Modern Animation, Movie Recording Technology, China}

\section{Introduction}

The development of the modern Chinese animation film is not only the imaging process of constant reform and innovation, but also a recording from the text alternative to the process of evolution of stereo sound. Studying modern Chinese animation film recording technology aims to promote the development of Chinese animation movie on the recording technology of pioneering and enterprising spirit. Through different historical periods Chinese animation recording technology of situation representation shows how Chinese animation film is first blockaded by foreign counterparts technology and animated movie and how Chinese animation film is self-reliant to break from the difficult position, after continuous research and development and application of new technology and new way (Bai, 2013), introducing the process of animation film with Chinese characteristics to the world.

Animation movie recording technology development and live-action film, originally started with silent films. At that time, when need to animation actors speak, can temporarily switch screen, the text displayed on the screen, and then switch back to the screen to continue. Silent film era, need animation characters speak, will be 
like a fish mouth spit bubbles, pop up a dialog, the dialog box shown in the figure to say. The traditional comic book character to speak in the same way. As for the background music, it will be in the movie theater in the form of a live band. Thus, original animation movie recording actually replaces the voice with words. To the 1920s after the tape recorder, a complete set of recording technology arises at the historic moment; this is a magnetic recording. The potential of this new technology in the film industry is immediately captured by sensitive practitioners, and then put into film production, but the technology is still the original; the operation method is only in bed playing records at the same time; the original band is replaced by a machine (Sun, 2014). Although in today's view this model is original and backward, it still marks a film from the silent movie era to era of sound.

\section{The First Recorded Animation Film in China}

\subsection{The First Two Movie Recording Modes}

The world's first two movie recording mode is of the 1930s "Vitaphone" and "Movietone". The former is the movie sound recorded on the record; the latter will be directly recorded on film sound. "Vitaphone" recording mode has an advantage when filming usually take more machines at the same time, this is to ensure the picture and sound synchronization operation, avoid appearing in the middle of the two lenses again voice "card" or "jump" problem. But its shortcomings also do not allow to ignore, the camera number determines the flight reservation, so the length of the lens cannot arbitrarily shortened, which makes the pride of the photography, namely the use of "montage". In the end "Vitaphone" in 1930. "Vitaphone" due to the recorded on film sound at the same time, there is no camera number of slots and the influence of the length of the lens, so "Vitaphone" after "Vitaphone" are widely accepted in the studios of recording technology.

\subsection{China's First Recorded Animation Film "Camel Offer Dance"}

In 1935, star film company hired well-known filmmakers WJH brother preparing first recorded in the history of Chinese animation film "camel offer dance". At this point, the film production and technology of China not only lagged behind the west, and is blocked by western filming technology of troubles. WJH brothers with the wisdom of the Chinese filmmakers and adhere to break through the various technical difficulties, created the group by means of experiment with shooting the tape after synthetic stereo recording mode, perfect solved the "camel offer dance" in the most difficult a lens "laughter". "Camel offer dance" success laid the foundation of Chinese audio animation films, making Chinese audio animation film in the extremely difficult and competitive in the market to survive.

\section{Recording and Early Late}

\subsection{Post Recording}

Late recording is simple lip-synching dubbing, first appeared in the late 1920s early 30s, when MGM film need to shoot a few scenes, in order to catch time and save cost, film companies simply let the actors in the film characters of mouth say change with lines, only to find meaning, started late recording (voice). Until today, our country most TV animation in adopting this recording mode. After the completion of the recording is late in the movie clips produced equals the film music, follow the rhythm of the movie and then let the band playing, just with the computer technology, modern late recording has been changed to digital music synthesis. Late dubbing is clipped a speaking part in the movie, the actor according to the characters in the movie mouth changes than read his lines. But this kind of mode for audio animation film is a difficult problem, is the mouth of no late dubbing often happen, animation film pay attention to the height of the picture and sound synchronization, contagious film effect can be attained. Great direct consequence is out of sync to animation film negative impact on the overall look and feel.

\subsection{Pre-Recording}

To solve the problem of recording of late, with the first recording arises at the historic moment. Early recording and later on the contrary, recording voice first, draw the picture. This pattern of voice actors capability demand is higher, good actor able to shape the role, with sound for masters of animation, good actors expressive voice 
acting is the source of painters inspiration, with sound painting pictures, not only solved the problem by synching up, and the creation of animated characters more full of vitality. Our country adopts the model of early recording animated film representative works include "the reed pipe", "three monks", "lotus lantern".

\section{The Influence of the Noise}

The presence of noise for film has always been an unavoidable and difficult to solve big problems, environmental noise and noise equipment, are in the process of film recording actual need sound interference by film. Early in the animated film, the noise is deadly "indifference", was used widely in optical cords every transcription, sound quality will be a lot of loss, this kind of noise for the recording medium itself quality reasons must be covered by powerful background music, or movies can't play properly. Modern audiences watch traditional animation film slightly look to the you will find that $\mathrm{s}$ animated films are instruments of pronunciation instead of the sound in real situation, such as the furious collision objects or role was hit by the violence weights, the audience heard is percussion stress, the most typical representative is welcomed by the audience favorite Disney old Tom and Jerry, when Tom was Jerry often play was struck or collision, background music is the sound of drums in different instrument parts.

Similarly, "reed" made in China in 1962 is adopted the same way. The reed pipe with pure Chinese style at the head of the film, not only pictures are all traditional Chinese ink painting, background music is also the Chinese traditional ethnic Musical Instruments, flute. The whole movie didn't show up line, the only sound is the melodious flute. With the picture of mist, bamboo flute pathetic, dreaming, audience, intoxicated.

Although the optical quality of the vocal cords over time and a great progress has been made in technological progress, but the noise is always exist. Animation film sound cannot replace all to music, meet characters must speak, problems arise. Due to the background noise is too strong, voice actors had to raise volume NianBai loudly to swamp the noise interference, this makes the voice acting is hard. China's 1941 animated film "princess iron fan" is the problem. Despite the publicity stunt movie is "the first recorded three-dimensional cartoons", but in today's audience, actors as if to deduce stage, almost every word is stressed, and the pronunciation all rise, seems to be no matter what kind of identity, role is to be screaming in an effort to show. The dubbing is the result of the drama almost into comedy, was serious and even tense scenes in the audience was very happy to feel, to laugh almost.

The noise magnetic powder until the 1950s after the film was basically solved, this to besmear brushs magnetic powder forming magnetic film sound track wider frequency response, letter voice than obvious than in the past, can not only ensure quality of voice, and recorded and transcribed the operation is simple, and can reproduce the recording as a result, the instant than traditional optical soundtrack have a qualitative leap. The invention of the magnetic film dubbing finally returned to normal vocal range, at the same time, the more subtle sound in the natural environment can be easily recorded by the media, 1960 animated film "little tadpole looking for mom" is used the film copy, little tadpoles childish naive voice and frog tenderness as the voice of effects between mother and let the audience feel the closeness of precious affection.

On the end of the world, the emergence of television formed a huge impact on the development of film, coupled with the growing popularity of the stereo home theater systems, film again to get the noise and its related practitioners, and bred for film and theater Dolby noise reduction technology, make the movie recording technology up to a new level (Rong, 2002).

\section{The Application of Stereo}

\section{1. "Magic Sound" System}

Stereo in early November 1940 Disney animated film "fantasia" have appeared, the technical personnel in the middle of the traditional speakers each side added a speaker, formed three channel effects, animation at this time also in the use of optical soundtrack recording medium, keep in sync with film screening, the sound recording and playback mode called "magic sound" system. However "magic sound" as much as seven tons of dead weight and expensive price keeps it from being widely spread in each big cinema. With the outbreak of World War II, the system gradually fades out the line of sight of people.

\subsection{Dolby Noise Reduction}

This is in the 1970s by Dolby stereo playback sound system, mainly for film and theater sound recording and 
playback. Dolby system USES optical soundtrack is still mode, great production at the same time, low cost, good effect, become the ancestor of the later digital recording technology and standards. In the 1980s, China's film industry started using Dolby A and SR analog stereo system. To the late ninety s began to upgrade for the SR digital stereo system and DTS system. First use this advanced sound system, due to insufficient skills in technology, also cannot give full play to its seismic effect.

When filming "lotus lantern", despite the use of the most advanced recording and DTS system, are still not satisfactory in many places. For example, opened a scene, with the decomposition of cracking of Huashan mountain, mountain building collapsed, it should be a shaking, thrilling movie special effects scene, still can be the final finished product a more thin and failed to generate sound coming from all directions, almost will be surrounded by people, the effect of final producers have to use the traditional electronic instrumental music sound to cover. In addition, in the celestial war scene, aloes, and Er Lang god absolute being in heaven, and two people in the mist, though not words, but the air is filled with a thick strong, at this moment the acoustics should build an occasion, the tense atmosphere, can be only background music in the film a sideshow elements, lead to the threat of war tension reduced many, is very regrettable thing.

As domestic filmmakers to more and more skills in the SR and DTS system, similar to the "lotus lantern" sound problem during production have been fully resolved. Theaters since in most regions are still mainly use the two stereo playback mode. Due to the cost problem, the current has not been fully used in domestic theaters digital stereo system, part of the theater still using analog stereo. As the technology progress and cost reduction, the future Chinese cinemas will adopt digital stereo sound playback mode, it will greatly improve the quality of the film's sound effects and watch.

\subsection{Surround Sound}

This is on the basis of Dolby SR and DTS upgrade version, in addition to the existing left and right surround sound added in around and around, around the viewer can create a more the voice of the real scene. Application of this system can accurately distinguish the audience in the direction of movement of the sound. Once upon a time, for example, when the rear direction of movement, the human ear can accurately judge what is behind the move to the left or the right rear. The technology performance in fast moving objects or action can result in more vivid sound effects.

\section{Conclusion}

In animated cartoon, sometimes actors create a character by bringing a distinct personality to the role, and to request seriously by synching up one film is higher. Modern Chinese animation film recording technology has experienced the silent film era, under the tireless efforts of generations of filmmakers have made substantial progress. With technology development, the Chinese animation film production levels are rising. Under the impetus of the modern computer and network technology, the Chinese animation film recording technology is to catch up with the international advanced level, forming its own unique style.

\section{References}

Bai, Z. Y. (2013). Discuss about the Origin and Development of Modern Chinese Animation. LanTai Word, 31, 118-119.

Rong, T. (2002). Chinese Movie Industrialization Problems. Nankai Management Review, 5, 71-74.

Sun, D. L. (2014). The Production of Animation Film Sound Is Analysed. The Home of the Drama, 17, 168. 